\title{
Impacto del programa de alfabetización informacional en la Universidad de las Ciencias Informáticas
}

\author{
Liuris Rodríguez Castilla \\ Universidad de las Ciencias Informáticas - UCI, Cuba
}

CASE REPORT

\begin{abstract}
Resumen
La investigación describe la evaluación y el impacto de la Alfabetización Informacional en la Universidad de las Ciencias Informáticas, a partir de la aplicación de un programa de formación en cursos de posgrado. Para la obtención de los resultados se utilizaron las técnicas de la encuesta, el análisis estadístico y la constatación, seleccionando una muestra de 84 involucrados en los cursos, clasificados en egresados y capacitadores. La evaluación de los resultados se divide en dos etapas: antes y después de impartir el programa de alfabetización informacional, a partir de un conjunto de indicadores. Los cursos han tenido gran aceptación en la universidad, considerándose muy útil y necesario tanto para estudiantes como para capacitadores.
\end{abstract}

Palabras clave

Alfabetización informacional ; Evaluación ; Posgraduación ; Universidad de las Ciencias Informáticas ; Cuba

\section{Impact of information literacy program in the Universidad de las Ciencias Informáticas}

\begin{abstract}
The research describes the evaluation and impact of Information Literacy through the implementation of a training program in postgraduate courses at the Informatics Science University. To obtain the results were used survey techniques, statistical analysis and verification, selecting a sample of 84 trainees involved in the courses, ranked graduates and trainers. The evaluation of the results is divided into two steps: before and after the course has been taught. A set of indicators have been taken. The courses have been widely accepted and considered very useful and necessary for both students and trainers at the university.
\end{abstract}

Keywords

Information literacy ; Evaluation; Posgraduate ; Universidad de las Ciencias Informáticas ; Cuba

\section{Introducción}

El desarrollo de las tecnologías computacionales y de la informática, así como el acceso a la información a través de redes e internet, son algunos de los medios cada vez más usados en la educación superior por estudiantes y profesores para su superación diaria. El uso de estas tecnologías, incrementa su aprendizaje acelerado como una herramienta de comunicación que permite el intercambio, surgimiento y socialización de información. Todo esto ha dado lugar a un crecimiento exponencial de nuevos conocimientos, así como la introducción de nuevas materias en la enseñanza, que permitan adquirir destrezas para interactuar con el software y hardware de estos equipamientos tecnológicos.

Una de estas materias surgidas a partir del uso de las tecnologías, es la Alfabetización Informacional (ALFIN), que comenzó a tomar auge a partir de los años 70 respondiendo a las perspectivas de nuevos espacios en las sociedades. Pero la Alfabetización Informacional centra su atención en la enseñanza para identificar, localizar, 
acceder, organizar, seleccionar, diseminar, usar de manera ética y socializar la información a través de estas tecnologías.

Esta disciplina ha sido aplicada en el sector educativo y de investigación de disímiles países como Estados Unidos (New York, Florida, Washington), España, Portugal, Francia, Suecia, Asia (China-Universidad de Tsingtao), Australia, Reino Unido, Irlanda, región oeste de África (Gana), Jamaica y en países latinoamericanos como México, Colombia, Perú, Cuba y otros. El apoyo de varias organizaciones ha sido clave para su avance, dentro de las que cabe destacar la UNESCO, ALA, IFLA, ALFINCAT, ACRL, ANZIIL, FUNDACIÓN GABRIEL PIEDRAHITA, esta rama del conocimiento alcanza cada vez más auge en el sector educativo y se impone ante los nuevos retos tecnológicos. En la actualidad, la temática cuenta con una red de colaboradores en el mundo que desarrollan programas, normas y estándares aplicados a sus sistemas educacionales para la instrucción de estos nuevos conocimientos (Uribe Tirado, 2010).

En Cuba han sido aislados los esfuerzos por diseminar y aplicar estos conocimientos, sin embargo a partir del 2005 ha tomado más fuerza en algunas universidades como: Universidad de la Habana (UH), Centro Universitario José Antonio Echevarría (CUJAE), Universidad Central de las Villas (UCLV), Universidad de Matanzas, Universidad de las Ciencias Informáticas (UCI), Facultades de Ciencias Médicas (INFOMED), Centro Nacional de Derecho de Autor, Instituto de Neurología y Neurocirugía de La Habana. Otros avances de han reflejado en investigaciones de grado, maestrías y doctorados destacándose autores como: (Lahera, 2002); (González, 2004); (Santos, 2004); (L. R. V. Valdés, 2005); (Medina, 2005); (Y. R. Valdés \& González, 2006); (Placeres, 2006); (Rodríguez Castilla, 2007b)

\section{Situación problemática}

En el ámbito profesional, toda investigación, proyecto a desarrollar, nuevo producto, artículo a publicar, etc., lleva implícito el uso de la información desde varias aristas:

- Se requiere de una búsqueda de información que corrobore los antecedentes o marco teórico referencial de los resultados que se desean obtener.

- Para la búsqueda de esta información en necesario tener definido qué se busca y cómo se desean los resultados.

- Se requiere de fuentes confiables, de información seleccionada y evaluada por especialistas e investigadores según su relevancia.

- Para buscar en las fuentes de información, se emplean estrategias de búsqueda.

- Para la organización y cita de la información encontrada, se requiere del dominio de manejadores bibliográficos.

- Para publicar los resultados de los trabajos adecuadamente se debe tener cultura científica e investigativa.

Es por ello que cualquier persona del ámbito académico, científico, investigativo, que desee alcanzar cultura en el trabajo con la información como superación profesional, debe contar con conocimientos básicos que les permita buscar, recuperar, organizar y evaluar la información necesaria para el desarrollo de sus proyectos, investigaciones, artículos, tesis, etc. La alfabetización informacional constituye la vía fundamental para que adquieran estos conocimientos.

Por otra parte, en los programas de estudios muchas universidades, se imparten materias sobre metodología de la investigación, donde el estudiante adquiere conocimientos básicos para llevar a cabo una investigación científica, concluyendo en la mayoría de los casos con un trabajo de diploma. Algunos de los temas abordados en esta materia son: la revisión bibliográfica, conformación del estado del arte, la redacción del marco teórico referencial y la cita de referencias bibliográficas. Pero estos contenidos, muy necesarios, resultan difíciles y trabajosos para el estudiante cuando se llevan a la práctica, e incluso, para muchos investigadores después de graduados. 
Requiere de un trabajo largo y exhaustivo al que hay que dedicarle buena parte del tiempo en la investigación y para el que no siempre se facilitan técnicas o herramientas que ayuden a agilizar o hacer más viable el proyecto. Para los diplomantes realizar el "capítulo teórico" de una investigación, resulta una tarea de gran envergadura y aunque cuentan con tutores que los guíen y reciben una materia que aborda estos temas, constantemente se hacen preguntas como estas:

¿Qué antecedentes revisar? ¿Por qué es importante analizar en la literatura lo que se ha escrito sobre el tema?, ¿Cómo hago una búsqueda? ¿Qué términos empleo?, ¿Cómo acceder a esa información? ¿Quiénes pueden ser los autores más relevantes en el tema que voy a estudiar? ¿Hasta dónde debo revisar? ¿Dónde puedo buscar información? ¿Qué puede ser lo más importante? ¿Cómo saber si la información que encuentro es confiable o no? ¿Qué es la bibliografía? ¿Qué una cita bibliográfica? ¿Qué es una norma bibliográfica? ¿Cómo hacer las referencias bibliográficas?

Una vez concluidos sus estudios universitarios, los egresados se quedan con lagunas en el ámbito de la investigación científica. Solo un pequeño por ciento muestra interés en dar a conocer los resultados de su investigación, pues no cuentan con cultura para la publicación de artículos en revistas académicas y científicas. Todas estas investigaciones, en buen número de gran relevancia, se queda solo en la literatura gris de las facultades donde estudiaron, ya que publicar o socializar la información y el conocimiento que generaron, lo ven como un trabajo tan complicado como volver a realizar "la tesis". Entonces surgen nuevas interrogantes.

¿Dónde puedo publicar? ¿Qué revistas abordan temas relacionados con mi investigación? ¿Qué revista escojo? ¿Cuál es la mejor? ¿Lo puedo publicar en Internet? ¿Dónde? ¿Cómo publicar en un sitio? ¿Cuánta gente ve mi trabajo? ¿Cómo escribo un artículo? ¿Qué son las normas de publicación? ¿Tengo que volver a hacer la bibliografía por otra norma?

Es por ello que a partir de estos problemas identificados, en la Universidad de las Ciencias Informáticas (UCI) se comienzan a desarrollar acciones orientadas para alfabetizar informacionalmente a los estudiantes, investigadores, profesores, especialistas y toda su comunidad universitaria, contribuyendo con ello a garantizar una adecuada investigación bibliográfica en los trabajos de diploma y una base sólida como investigadores en su vida profesional. Una de estas actividades es la capacitación a través de curso de posgrado que apoya la enseñanza de la Metodología de la Investigación y fortalece la integración de los procesos docencia-investigación-producción.

El objetivo de esta investigación de dar a conocer los resultados obtenidos en la aplicación de estos cursos de posgrado y el impacto que ha tenido en la Universidad de las Ciencias informáticas.

\section{Materiales y métodos}

Para evaluar el impacto que ha tenido la implementación de los cursos de posgrado en la UCI, se utilizó el apoyo de la Dirección de Posgrado de la Universidad, solicitando la cantidad de cursos ofrecidos, los capacitadores y los egresados de cada curso. Se encontraron un total de 32 cursos dados entre 2005 y 2012 por 17 capacitadores, con un total de 437 estudiantes graduados

Se utilizó la técnica de la encuesta y el análisis estadístico para la recogida de información, tanto para capacitadores como los alumnos egresados del curso.

Del total de egresados de los cursos de posgrados (437), 109 ya no son usuarios de la UCl, contando solamente en la Universidad con $\mathbf{3 2 8}$ para la investigación. Para ello se seleccionó una muestra de $\mathbf{1 0 0}$ graduados para encuestar, de los cuales respondieron 77. De los capacitados se seleccionó una muestra de 10 de los cuales respondieron 7.

El análisis de los resultados se llevó a cabo a través del método de la constatación. Se dividió la muestra en dos grupos: estudiantes de posgrados (egresados de los cursos) y capacitadores. Al grupo de los estudiantes le aplicó un conjunto de indicadores y sub-indicadores para evaluar sus conocimientos sobre alfabetización informacional en dos etapas, antes de recibir el curso y después de recibirlo. Para el grupo de los capacitadores lo indicadores estuvieron relacionados con las características para impartir los cursos y las evaluaciones generales de cada uno. 


\section{Resultados y discusión}

Como antecedentes de este estudio, se realizó una investigación de maestría (Rodríguez Castilla \& Torricella Morales, 2008) en el que se propuso un Programa de Alfabetización Informacional a líderes y equipos de proyectos informáticos, a partir de una metodología de desarrollo del software (RUP). Este programa ofrecía la posibilidad de adaptarlo a otros entornos y se elaboró una versión para cursos de postgrado de forma general. Para ello se analizó los posibles elementos de apoyo dentro de la UCl que permitieran la viabilidad del aprendizaje de ALFIN.

\subsection{Apoyo de la Universidad}

- Asignatura Práctica Profesional a estudiantes de 1er año, en el que se abordan varios temas de manera integradoras de ALFIN.

- Jornadas científicas estudiantiles, con la presentación trabajos de investigaciones que tributen a su desempeño docente- productivo.

- La Dirección de Posgrado promueve cursos de superación profesional sobre Alfabetización Informacional con mayor profundidad de contenidos que los de pregrado, conocidos como Infotecnología y Alfabetización Informacional respectivamente.

- La Dirección de Información de la Universidad (Biblioteca) facilita a los usuarios, el acceso al catálogo en línea, bases de datos bibliográficas y otros recursos web de calidad científica y académica.

- La Dirección de Investigaciones por medio de la Editorial Universitaria, promueve la publicación de artículos o investigaciones terminadas a través del repositorio de la Serie científica y la Revista Cubana de Ciencias Informáticas, propia de la Universidad que aborda temáticas de las Ciencias Informáticas.

\subsection{El programa de Alfabetización Informacional aplicado.}

El programa describe los objetivos generales, objetivos Instructivos y objetivos instructivos. Es está estructurado con un total de 9 temas a impartir en 10 encuentros presenciales, estos pueden ser ajustados por el profesor en menos frecuencias presenciales, en función de las horas disponibles para cada tema (Rodríguez Castilla, 2012)

Tabla 1. Estructura del Programa de ALFIN de la UCI

\begin{tabular}{|l|c|c|c|c|c|c|}
\hline & CONFERENCIAS & CTP & TALLER & $\begin{array}{c}\text { ACTIVIDADES NO } \\
\text { PRESENCIALES }\end{array}$ & EVALUACIÓN & TOTAL \\
\hline ACTIVIDADES & 9 & 6 & 8 & 9 & 1 & 33 \\
\hline HORAS & 18 & 12 & 8 & 120 & 2 & 160 \\
\hline
\end{tabular}

*Nota: El programa esta conformado por nueve (9) contenidos temáticos.

Cada tema en el programa tiene definido los objetivos, el sistema de conocimientos, sistema de habilidades, la modalidad, las tareas a desarrollar y los materiales de apoyo. Los temas que contiene son:

- Tema I: La información como recurso.

- Tema II: Recursos y herramientas para la búsqueda y recuperación de información en Internet. 
- Tema III: Bases de datos bibliográficas.

- Tema IV: Selección y evaluación de la información.

- Tema V: Interpretación de referencias bibliográficas y nociones sobre formatos bibliográficos. Gestores Bibliográficos

- Tema VI: Evaluación y selección de información.

- Tema VII: Monitoreo de información y vigilancia tecnológica.

- Tema VIII: Principios metodológicos. Estado del arte y marco teórico referencial.

- Tema IX: Diseminación de conocimientos, socialización y publicación de información.

En el programa también se describen, las indicaciones metodológicas y de organización del curso, las condiciones generales, el sistema de evaluación. Cuenta además con una multimedia (Rodríguez Castilla, 2007a) y una versión en el Entorno Virtual de Aprendizaje de la Universidad (EVA (Rodríguez Castilla, 2013) como material de apoyo complementario tanto para capacitadores como para los estudiantes, en la que se incluyen todas las conferencias de cada tema, videos tutoriales, materiales bibliográficos a texto completo para su estudio independiente, instaladores de las herramientas a usar durante el curso, así como la bibliografía básica del curso.

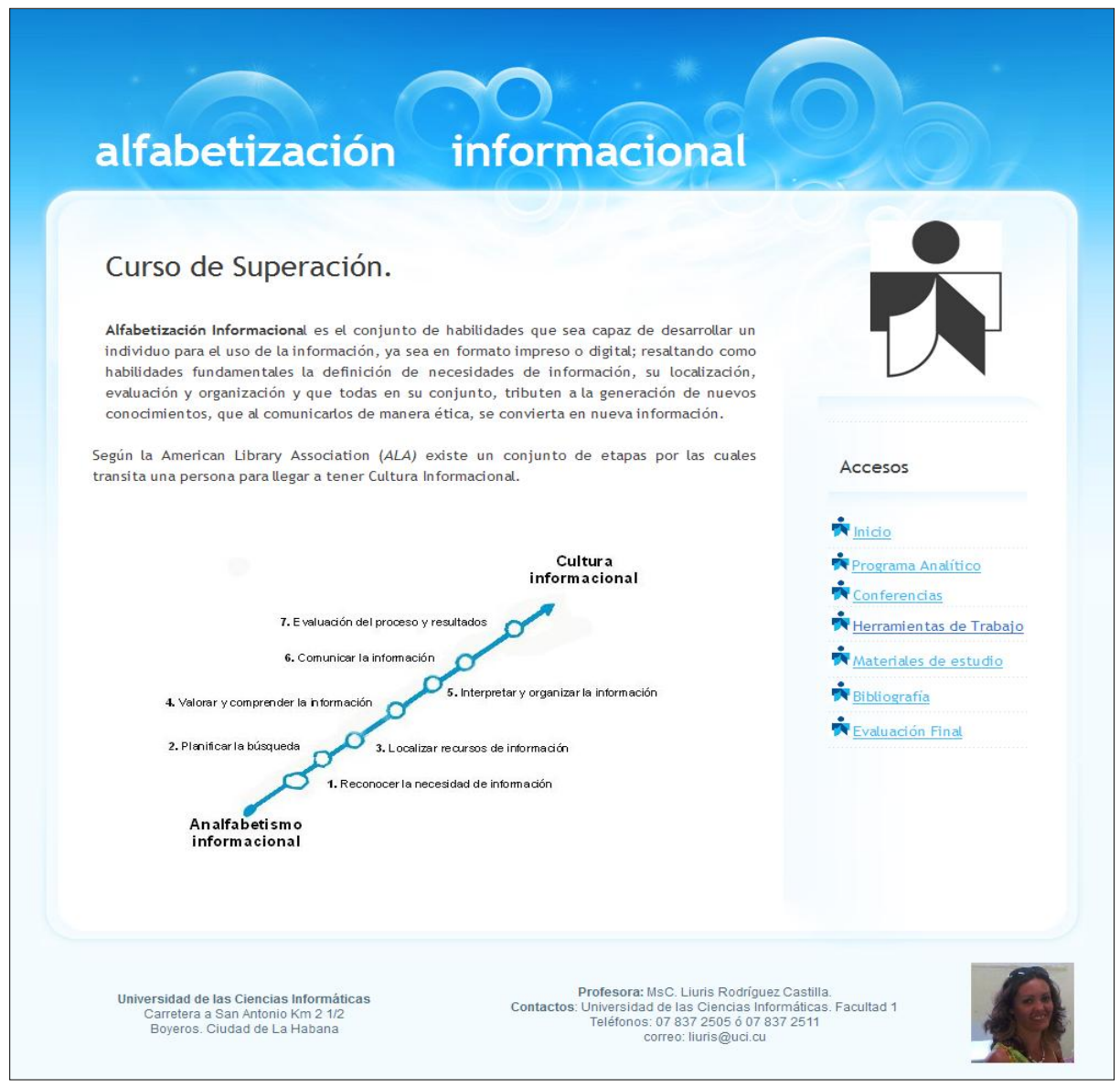

Imagen 1. Interfaz de la Multimedia de ALFIN 


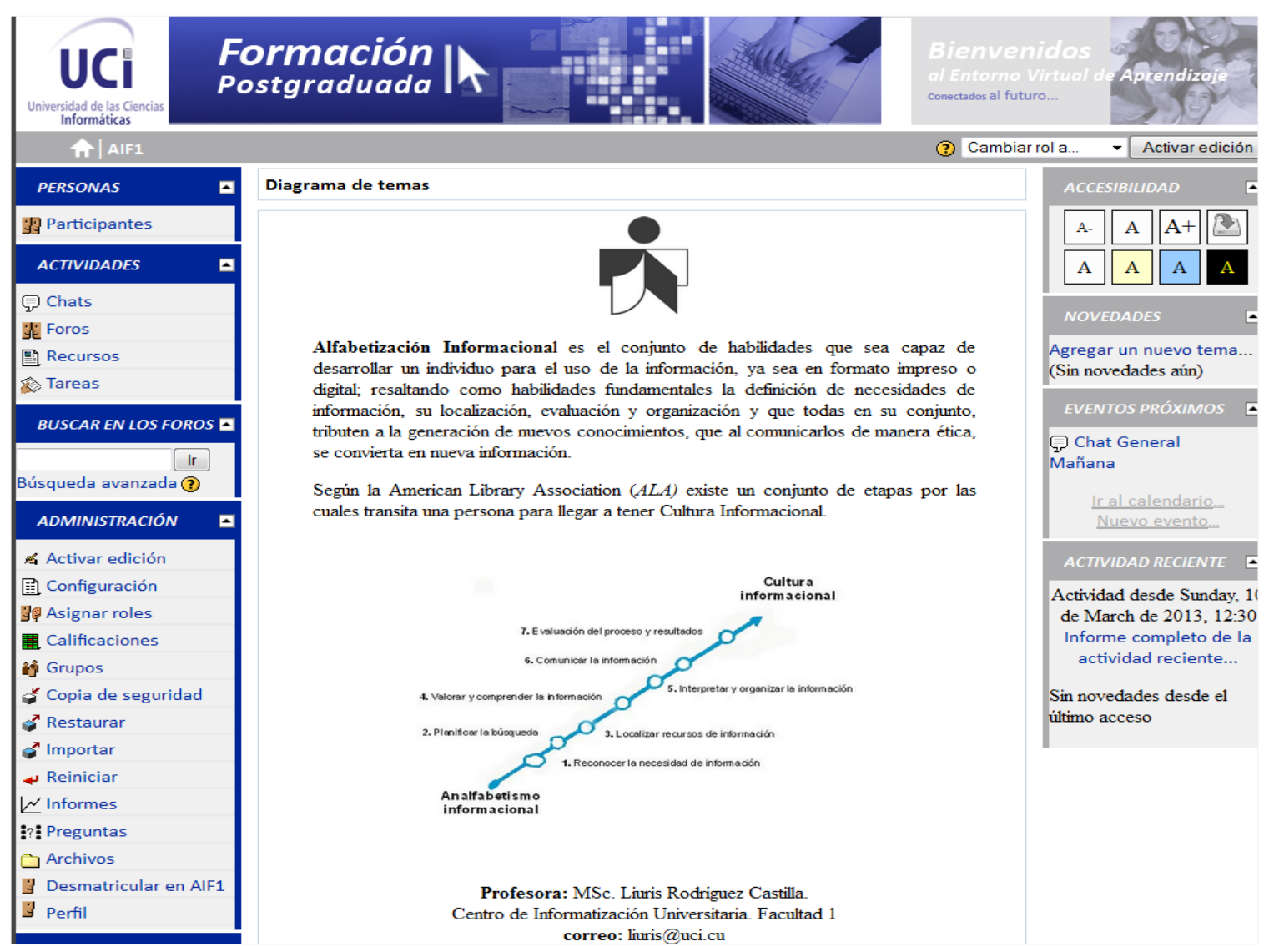

Imagen 2. Interfaz del curso en el EVA

El programa comenzó a impartirse en el 2005 a partir de un taller para capacitadores ofrecido por el Ministerio de Educación Superior de Cuba. Hasta el momento se han realizado 32 ediciones del curso bajo los nombres de Infotecnología y Alfabetización Informacional.

Tabla 2. Total de Cursos de posgrado impartidos en la UCl

\begin{tabular}{|c|c|c|}
\hline AÑO & CANTIDAD DE CURSOS & CANTIDAD DE EGRESADOS \\
\hline 2005 & 3 & 125 \\
\hline 2006 & 10 & 34 \\
\hline 2007 & 4 & 17 \\
\hline 2008 & 1 & 40 \\
\hline 2009 & 3 & 39 \\
\hline 2010 & 3 & 49 \\
\hline 2011 & 3 & 58 \\
\hline 2012 & 5 & 437 \\
\hline Total & 32 & \\
\hline
\end{tabular}


a) Resultados de los indicadores aplicados. Estudiantes egresados

Tabla 3. Evaluación de egresados

\begin{tabular}{|c|c|c|c|c|c|}
\hline \multirow{2}{*}{ INDICADOR } & \multirow{2}{*}{ SUB-IBDICADOR } & \multicolumn{4}{|c|}{ RESULTADOS } \\
\hline & & Antes & $\% 77$ & Después & $\% 77$ \\
\hline \multirow{2}{*}{ Conocimientos de ALFIN } & Definición & 6 & $8 \%$ & 68 & $88 \%$ \\
\hline & Aplicación & 2 & $3 \%$ & 65 & $84 \%$ \\
\hline \multirow{5}{*}{$\begin{array}{l}\text { Fuentes utilizadas para la } \\
\text { búsqueda de información }\end{array}$} & $\begin{array}{l}\text { Herramientas de internet (buscadores, guías } \\
\text { de materia, bases de datos) }\end{array}$ & 22 & $29 \%$ & 75 & $97 \%$ \\
\hline & Bibliotecas y centros de información & 12 & $16 \%$ & 40 & $52 \%$ \\
\hline & Expertos en el tema & 4 & $5 \%$ & 47 & $61 \%$ \\
\hline & Trabajos o materiales prestados & 44 & $57 \%$ & 10 & $13 \%$ \\
\hline & Lo primero que encuentre & 70 & $90 \%$ & 2 & $3 \%$ \\
\hline \multirow{8}{*}{ Cómo realizaban las búsquedas } & Por palabras clave & 59 & $77 \%$ & 70 & $90 \%$ \\
\hline & Búsqueda avanzada & 20 & $26 \%$ & 56 & $73 \%$ \\
\hline & Temáticas generales y específicas & 11 & $14 \%$ & 46 & $60 \%$ \\
\hline & Autores & 6 & $8 \%$ & 49 & $64 \%$ \\
\hline & Ayuda de las herramientas & 2 & $3 \%$ & 26 & $34 \%$ \\
\hline & Servicios que brindan las fuentes & 2 & $3 \%$ & 21 & $27 \%$ \\
\hline & Colecciones o grupos de fuentes & 1 & $1 \%$ & 16 & $21 \%$ \\
\hline & Cambian la estrategia de búsqueda & 3 & $4 \%$ & 40 & $52 \%$ \\
\hline \multirow{10}{*}{$\begin{array}{l}\text { Indicadores para seleccionar la } \\
\text { información }\end{array}$} & Autor & 5 & $6 \%$ & 54 & $70 \%$ \\
\hline & Prestigio de la fuente & 10 & $13 \%$ & 40 & $52 \%$ \\
\hline & Actualidad & 8 & $10 \%$ & 45 & $58 \%$ \\
\hline & Fecha de creación y publicación & 2 & $3 \%$ & 38 & $49 \%$ \\
\hline & Contenido de la información & 50 & $65 \%$ & 75 & $97 \%$ \\
\hline & Pertinencia & 2 & $3 \%$ & 30 & $39 \%$ \\
\hline & Relevancia & 4 & $5 \%$ & 31 & $40 \%$ \\
\hline & Avalada por expertos & 2 & $3 \%$ & 35 & $45 \%$ \\
\hline & El idioma & 30 & $39 \%$ & 72 & $93 \%$ \\
\hline & Declaración de principios del sitio & 0 & $0 \%$ & 19 & $25 \%$ \\
\hline $\begin{array}{l}\text { Citar las referencias } \\
\text { bibliográficas }\end{array}$ & Citan las fuentes & 19 & $25 \%$ & 76 & $98 \%$ \\
\hline \multirow{3}{*}{$\begin{array}{l}\text { Elaboración y organización de la } \\
\text { bibliografía }\end{array}$} & Gestores bibliográficos & 10 & $13 \%$ & 72 & $93 \%$ \\
\hline & Norma bibliográfica (manual) & 6 & $8 \%$ & 47 & $61 \%$ \\
\hline & Ejemplos ya hechos & 3 & $4 \%$ & 15 & $19 \%$ \\
\hline \multirow{4}{*}{$\begin{array}{l}\text { Publicación de sus } \\
\text { investigaciones }\end{array}$} & Eventos & 35 & $45 \%$ & 69 & $89 \%$ \\
\hline & $\begin{array}{l}\text { Serie científica (Revista digital propia de la } \\
\text { Universidad) }\end{array}$ & 12 & $16 \%$ & 35 & $45 \%$ \\
\hline & Revistas académicas o científicas & 3 & $4 \%$ & 19 & $25 \%$ \\
\hline & Sitios web o repositorios & 2 & $3 \%$ & 5 & $6 \%$ \\
\hline
\end{tabular}


b) Valoraciones generales de los estudiantes sobre el curso de posgrado.

Tabla 4. Valoraciones generales de los estudiantes

\begin{tabular}{|l|c|c|}
\hline \multicolumn{1}{|c|}{ Valoraciones generales } & N Estudiantes & 54 \\
\hline Afirman que se necesita más práctica & 46 & 19.01 \\
\hline Entienden que se debe extender en más clases & 38 & 32 \\
\hline Coinciden con más ediciones del curso & 31 & 13.38 \\
\hline Creen que se pueden incorporar nuevos contenidos & 20 \\
\hline $\begin{array}{l}\text { Profundizar más en los temas de publicación de artículos y la evaluación y calidad de la } \\
\text { información }\end{array}$ & 11.27 \\
\hline $\begin{array}{l}\text { Opinan que se incorporen otras formas de evaluación (publicación de un artículo, promedio } \\
\text { de las evaluaciones parciales, autodidacta) }\end{array}$ & 23 \\
\hline Sugieren convertirlo en un diplomado con mayor profundidad en los contenidos & 10.92 \\
\hline Opinan que se debe dar solo un tema por encuentro & 8.10 \\
\hline Posibilidad de usar más gestores bibliográficos en las clases & 6.34 \\
\hline Sugieren que se debe mejorar la multimedia & 7 & 2.46 \\
\hline
\end{tabular}

\section{c) Resultados de los indicadores aplicados. Capacitadores}

De los 17 capacitadores que han ofrecido el curso en la universidad como posgrado, 4 ya no son profesores en la $\mathrm{UCl}$, de los 13 restantes, se seleccionó una muestra de 10 capacitadores para realizar la investigación, respondiendo 7 la encuesta. Aunque todos capacitadores analizados habían sido profesores de posgrado, algunos también habían sido capacitadores en pregrado.

Tabla 5. Evaluación de capacitadores (continua)

\begin{tabular}{|l|l|c|}
\hline \multicolumn{1}{|c|}{ INDICADORES } & \multicolumn{1}{|c|}{ SUB-INDICADOR } & CANTIDAD \\
\hline \multirow{4}{*}{$\begin{array}{l}\text { Tipos de cursos } \\
\text { (Cantidad) }\end{array}$} & $\begin{array}{l}\text { Posgrado (Independientes- 7, Módulo de maestrías-doctorados-1 } \\
\text { A distancia -1) }\end{array}$ & 9 \\
\cline { 2 - 3 } & $\begin{array}{l}\text { Pregrado (1 ro-3er año-curso optativo- 4, 4to año-curso optativo- 3 } \\
\text { 5to año-curso optativo- 2) }\end{array}$ & 9 \\
\hline \multirow{3}{*}{$\begin{array}{l}\text { Formación como } \\
\text { capacitadores }\end{array}$} & A través de un curso de posgrado & 7 \\
\cline { 2 - 4 } & En un curso para capacitadores & 3 \\
\cline { 2 - 4 } & En preparaciones metodológicas de otras asignaturas & 2 \\
\cline { 2 - 4 } & Visitando la biblioteca de la escuela & 2 \\
\cline { 2 - 4 } & De forma autodidacta & 2 \\
\hline
\end{tabular}


Tabla 5. Evaluación de capacitadores (final)

\begin{tabular}{|c|c|c|}
\hline INDICADORES & SUB-INDICADOR & CANTIDAD \\
\hline \multirow{8}{*}{$\begin{array}{l}\text { Aspectos Positivos } \\
\text { de los cursos dados }\end{array}$} & Utilidad para toda la vida profesional & 7 \\
\hline & Actualidad en los temas & 5 \\
\hline & Apoyo a los procesos docente-investigativo-productivo & 5 \\
\hline & Flexibilidad para adaptarlo a disímiles contextos & 5 \\
\hline & Uso de la información en varios ámbitos & 5 \\
\hline & Relación con metodología de la investigación & 4 \\
\hline & Novedad en la materia & 3 \\
\hline & Vínculo de la teoría con la práctica & 7 \\
\hline \multirow{6}{*}{$\begin{array}{l}\text { Dificultades en los } \\
\text { cursos dados }\end{array}$} & Locales & 3 \\
\hline & Acceso a internet (conexión) & 5 \\
\hline & Entorno Virtual de Aprendizaje-redes & 4 \\
\hline & Catálogo la biblioteca & 3 \\
\hline & Falta de preparación personal & 2 \\
\hline & Pocos recursos de información & 2 \\
\hline \multirow{6}{*}{$\begin{array}{l}\text { Necesidades y } \\
\text { consideraciones } \\
\text { sobre los cursos } \\
\text { dados }\end{array}$} & Más capacitadores & 5 \\
\hline & Asignatura obligatoria & 8 \\
\hline & Preparaciones metodológicas para capacitadores & 6 \\
\hline & Sistematicidad en las ofertas de cursos & 6 \\
\hline & Nuevos contenidos & 4 \\
\hline & Más formas de evaluación & 3 \\
\hline $\begin{array}{l}\text { Necesario, útil y } \\
\text { atractivo }\end{array}$ & $\begin{array}{l}\text { Opiniones-Capacitadores } \\
\text { - En su preparación para la investigación, fundamentalmente para la realización de } \\
\text { sus tesis } \\
\text { - El curso relaciona el uso de la información científica para el ámbito académico y } \\
\text { científico, por lo que es una base muy necesaria para todo egresado universitario. } \\
\text { - Son nuevos conocimientos vinculados con las TIC por lo que les resulta muy } \\
\text { atractivo el hecho que se pueda llevar a la práctica. } \\
\text { - Creo que puede ser de muy buen provecho que todos los profesionales incorporen } \\
\text { estas habilidades a sus conocimientos. } \\
\text { - Casi siempre descubren durante el curso la importancia de la información y la } \\
\text { utilidad que le pueden dar, sobre todo cómo usar las fuentes de información. }\end{array}$ & 5 \\
\hline $\begin{array}{l}\text { Opiniones recogida } \\
\text { de los estudiantes } \\
\text { durante los cursos }\end{array}$ & $\begin{array}{l}\text { - "He sentido que he aprendido a hacer búsquedas en internet y organizar la } \\
\text { bibliografía". } \\
\text { - "Muy interesante porque aprendimos el alcance de internet, ya que solo } \\
\text { conocíamos los buscadores tradicionales, también adquirimos bases tanto para la } \\
\text { búsqueda de información como orientación para la publicación de sus artículos". } \\
\text { - "Curso muy bueno" } \\
\text { - "Pensé que sabía sobre este tema pero aprendí muchas cosas nuevas" } \\
\text { - "Creo que debe ser obligatorio para todos los profesores en la universidad" } \\
\text { - "Hace falta más práctica" } \\
\text { - "Debería certificarse el curso" }\end{array}$ & 7 \\
\hline
\end{tabular}


De los 7 formadores que respondieron la encuesta, solo 3 se mantiene actualmente como capacitadores, algunos de los motivos de aquellos que se retiraron fueron:

- " "No se ha concretado la próxima edición de cursos en la facultad donde trabajan".

- "En las facultades ese curso de modo optativo ya no se oferta"

- "Mucho contenido de trabajo como especialista y falta de preparación".

- "En ocasiones doy algún tema como complemento del curso de inteligencia empresarial, pues uno de los módulos es aprender a trabajar con las bases de datos y la web invisible de forma general, pero no doy el curso completo".

\subsection{Consideraciones finales}

Como se pudo observar, el curso ha tenido gran aceptación en la escuela, y se considera muy útil y necesario tanto para estudiantes como para capacitadores. A pesar de la mayoría de las opiniones positivas sobre las interrogantes realizadas en las encuestas, quedan muchos aspectos por mejorar para que el impacto de este cuso sea mayor y llegue a ser de interés en toda la comunidad universitaria.

1. Proponer la generalización del curso de forma obligatoria para todos los profesores al graduarse, como parte de su preparación inicial como adiestrados y como condicionante para el cambio de categoría docente.

2. Identificar posibles capacitadores y realizar varias ediciones de cursos para formar los diseminadores de estos conocimientos.

3. Establecer preparaciones metodológicas sistemáticas una vez preparados a los capacitadores.

4. Mantener actualizados los contenidos del curso y proponer nuevos temas que sean de interés, en la medida que de gane en experiencia con la edición de nuevos cursos y a partir de la sugerencia y demanda de los estudiantes.

5. Extender los encuentros presenciales, llevándolo a un tema por encuentro, que facilite mayor práctica para los alumnos.

6. Lograr profundizar en la publicación de artículos en revistas académicas y científicas, si dejar de presentar trabajos en eventos, pero que tenga mayor visibilidad en el web de la ciencia, los resultados investigativos de la Universidad.

7. Incrementar las ediciones del curso, pues la demanda de matrícula es alta.

8. Lograr que los alumnos que se matriculan terminen, ya que tiene mucha carga de trabajo docente que deben priorizar, y abandonan el curso. 


\section{Conclusiones}

- El uso de estas tecnologías, incrementa su aprendizaje acelerado como una herramienta de comunicación que permite el intercambio, surgimiento y socialización de información.

- La Alfabetización Informacional ha sido aplicada en el sector educativo y de investigación de disímiles países del mundo.

- La Universidad de las Ciencias Informáticas (UCI) se han venido desarrollando, acciones encaminadas a capacitación de los estudiantes, profesores e investigadores en ALFIN.

- Se encontraron en la UCl un total de 27 cursos de posgrados dados entre 2005 y 2011 por 17 capacitadores, un total de 397 estudiantes graduados.

- El curso ha tenido gran aceptación en la escuela, y se considera muy útil y necesario tanto para estudiantes como para capacitadores.

\section{Recomendaciones}

1. Proponer nuevas modalidades del curso (semi-presencial o a distancia)

2. Establecer talleres sobre temas específicos (Ej. Publicación de artículos) que sean de corta duración (1 día), utilizando esta vía para la promoción e incentivando a los interesados a matricular el curso completo.

3. Presentar ediciones especiales avanzadas para usuarios que ya hayan recibido el curso.

4. Proyectar el curso en una edición de Diplomado.

5. Proponer el curso como módulo de las maestrías y doctorados que se editen en la Universidad.

6. Publicar el resultado del la investigación sobre el impacto del curso en una revista académica o científica, ampliando la muestra para obtener mayores resultados.

\section{Bibliografía}

González, Y. R. (2004). Alfabetización informacional en los trabajadores sociales de la Sede Universitaria Municipal del MES de Plaza de la Revolución. Tesis para optar por el título académico de Licenciatura en Información Científico-Técnica y Bibliotecología Tesis de diploma, Universidad de La Habana, La Habana.

Lahera, Y. M. (2002). Cultura y Alfabetización informacional . Una aproximación a su estudio. Tesis para optar por el título académico de Licenciatura en Información Científico Técnica y Bibliotecología Tesis de diploma, Universidad de La Habana, La Habana.

Medina, Y. B. (2005). Alfabetización y comportamiento informacional . Estudio de caso. Tesis para optar por el título académico de Licenciatura en Bibliotecología y Ciencias de la Información Tesis de diploma, Universidad de La Habana, La Habana.

Placeres, G. M. (2006). Los programas de alfabetización Informacional en las universidades. Tesis para optar por el título académico de Máster en Bibliotecología y Ciencias de la Información Tesis de maestría, Universidad de La Habana, La Habana.

Rodríguez Castilla, L. (2007a). Multimedia "Alfabetización Informacional". La Habana: Universidad de las Ciencias Informáticas. 
Rodríguez Castilla, L. (2007b). Propuesta de un programa de Alfabetización Informacional para proyectos productivos en la Universidad de las Ciencias Informáticas. Maestría TESIS PARA OPTAR POR EL TÍTULO DE MÁSTER EN GESTIÓN DE PROYECTOS INFORMÁTICOS, Universidad de las Ciencias Informáticas, La Habana.

Rodríguez Castilla, L. (2012). Programa Analítico de un Curso de Postgrado "Alfabetización Informacional" (8 ed., pp. 17). La Habana: Vicerrectoria de Formación: Dirección de Formación Posgraduada. Universidad de las Ciencias Informátcas.

Rodríguez Castilla, L. (2013). Curso de Posgrado "Alfabetización Informacional " Retrieved marzo, 2013, from http://evapostgrado.uci.cu/course/view. php?id=358

Rodríguez Castilla, L., \& Torricella Morales, R. G. (2008). La Alfabetización Informacional en los procesos de desarrollo de software. Propuesta de un programa para la Universidad de las Ciencias Informáticas. Ciencias de la Información(3), 3-19.

Santos, I. D. d. I. C. (2004). Propuesta de alfabetización tecnológica en el Centro Nacional de Derecho de Autor. Tesis para optar por el título académico de Licenciatura en Bibliotecología y Ciencias de la Información Tesis de diploma, Universidad de La Habana, La Habana.

Uribe Tirado, A. (2010). La alfabetización informacional en Iberoamérica.

Valdés, L. R. V. (2005). Propuesta de un programa de alfabetización informacional para usuarios del Instituto de Neurología y Neurocirugía. Tesis para optar por el título académico de Máster en Bibliotecología y Ciencias de la Información Tesis de maestría, Universidad de La Habana, La Habana.

Valdés, Y. R., \& González, Y. C. (2006). Alfabetización Informacional: estudio de caso en la Facultad de Comunicación. Tesis para optar por el título académico de Licenciatura en Bibliotecología y Ciencias de la Información Tesis de diploma, Universidad de La Habana, La Habana.

\section{Datos de la autora}

\section{Liuris Rodríguez Castilla}

Profesora - Especialista en Información. Centro de Informatización Universitaria (CENIA). Universidad de las Ciencias Informáticas. Licencia en Información Científico Técnica y Bibliotecología en la Universidad de la Habana (2003), Máster en Gestión de Proyectos Informáticos (UCl-2007). Profesor asistente UCl. Ha cursado diversos estudios de posgrado nacional e internacional. Ha impartido más de 20 cursos de Posgrado Alfabetización Informacional y otros de Arquitectura de Información. Cuenta con varias publicaciones en revistas académicas y científicas como ACIMED y Ciencias de la Información y en las memorias de Eventos y Congresos respectivamente. Ha participado diversos eventos nacionales e internacionales.

\section{liurisz@gmail.com}

$\begin{array}{ll}\text { Recibido-Received } & : 2013-03-22 \\ \text { Aceptado-Accepted } & : 2013-12-30\end{array}$

\section{(cc) EY}

This work is licensed under a Creative Commons Attribution 4.0 United States License.

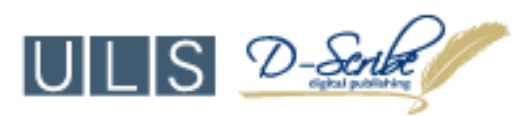

This journal is published by the University Library System of the University of Pittsburgh as part of its $\underline{D}$-Scribe Digital Publishing Program and is cosponsored by the University of Pittsburgh Press. 